Open Peer Commentary for Do Researchers Have an Obligation to Actively Look for Genetic Incidental Findings?

\title{
THE DUTY TO RESCUE IN GENOMIC RESEARCH
}

\author{
Michael Ulrich, J.D.
}

Gliwa et al. (2012) focus their analysis of researcher obligations for incidental findings in genomic research on three criteria: (1) the benefit to the research participant, which requires strong associations with severe disease that has medical actionability; (2) unique access to the information and analysis; and (3) the burden that looking and returning incidental findings places on the researcher. ${ }^{1}$ While the authors adopt the ancillary care framework to analyze requirements to return incidental findings, I believe the duty to rescue presents several advantages while preserving those benefits found in the ancillary care model. First, utilizing the duty to rescue avoids conflating the return of genetic information with providing needed clinical care. Second, a rescue-based approach recognizes the ethical duties researchers have toward the research study and offers a mechanism for appropriately balancing these with obligations to individual subjects. Finally, using the duty to rescue as the ethical underpinning for incidental finding obligations answers definitively that there is no duty to search for incidental findings.

The duty to rescue has been underanalyzed and relatively unexplored in recent debates on research ethics (Merritt, Taylor, and Mullany 2010). ${ }^{2}$ In terms of the incidental findings quandary, the duty to rescue model requires disclosure when a researcher discovers genetic information that indicates a risk of significant harm that they are in a position to mitigate with little risk to the overall research study. While there are some similarities between this approach and one anchored in the ancillary care framework, the duty to rescue presents a more definitive answer in what must be returned. For example, the authors contend that duties of ancillary care in genomic research relate to information that is medically beneficial rather than increasing knowledge or awareness (Gliwa et al. 2012). ${ }^{3}$ Yet, under an ancillary care analysis others have determined researchers are required to return incidental findings the subject is likely to believe

\footnotetext{
${ }^{1}$ Catherine Gliwa et al., Do Researchers Have an Obligation to Actively Look for Genetic Incidental Findings?, American Journal of Bioethics (2012): 6.

${ }^{2}$ Maria W. Merritt, Holly A. Taylor, \& Luke C. Mullany, "Ancillary Care in Community-Based Public Health Intervention Research,” American Journal of Public Health 100 (2010): 212.

${ }^{3}$ Gliwa et al. (2012): 12.
} 
are of health importance or reproductive importance (Wolf et al. 2008). ${ }^{4}$ Thus, a clear conflict arises in attempting to determine what qualifies as mandatory information to return when utilizing an ethical framework that was developed in the context of clinical care (Richardson and Belsky 2004). ${ }^{5}$

Conflating the provision of clinical care and genetic information may be dangerous given the manner in which our understanding of the connections between genetic variants and specific diseases is constantly evolving. Even the authors concede that genomic science is in its infancy and, therefore, cannot necessarily be described as a benefit in every circumstance (Gliwa et al. 2012). ${ }^{6}$ As such, it appears that distinguishing between supplying ancillary care and ancillary information may be helpful not only to IRBs and researchers but, more importantly, to subjects (Clayton and McGuire 2012). ${ }^{7}$ Indeed, merging these two paradigms may play a part in the often cited preferences of subjects to receive any and all information that can be gathered from their genomic sequencing. With the risk of a therapeutic misconception always looming, it is imperative that the possibility of receiving any genetic information not be equated to an opportunity to cure a disease that is certain to arise. Meanwhile, the duty to rescue informs all parties involved that only in the case where there is significant risk of harm that can be mitigated should incidental finding obligations begin to arise.

These duties to return incidental findings must be weighed against the burden such a duty places on the researcher, as Gliwa et al. (2012) repeatedly state. ${ }^{8}$ The ethical responsibilities that researchers have to the study itself, including the duties to achieve the study goals and to the subjects who enrolled in the study to help accomplish those goals, are often overlooked. While many subjects may have incidental findings, there may be other subjects who do not. The subjects who do not underwent risks of enrollment to help achieve the research objective and, therefore, the researcher has an obligation to see that the study protocol is followed to completion. It would certainly be an ethical paradox if requirements to return incidental findings

\footnotetext{
${ }^{4}$ Susan M. Wolf et al., "Managing Incidental Findings in Human Subjects Research: Analysis and Recommendations,” Journal of Law, Medicine \& Ethics 36 (2008): 15.

${ }^{5}$ Henry S. Richardson \& Leah Belsky, “The Ancillary-Care Responsibilities of Medical Researchers,” Hastings Center Report 34 (2004): 26.

${ }^{6}$ Gliwa et al., (2012): 14.

${ }^{7}$ Ellen Wright Clayton \& Amy L. McGuire, “The Legal Risks of Returning Results of Genomics Research,” Genetics in Medicine 14 (2012): 473.

${ }^{8}$ Gliwa et al., (2012): 6, 9, 12, 13-14, 17.
} 
to certain individual subjects became so broad as to demand too many resources to allow for the completion of the study purpose they supposedly enrolled for (Meyer 2008). ${ }^{9}$

The duty to rescue provides an inherent mechanism to appropriately balance the obligations to individual subjects with the overall research study. The duty to rescue arises when there is a risk of significant harm that the rescuer's action has a high probability of mitigating, there is little risk of harm to the rescuer, and the benefit gained outweighs the potential risks (Beauchamp and Childress 2001). ${ }^{10}$ That last aspect of balancing the benefits and the burdens ensures that the pursuit of generalizable knowledge maintains its place as the primary purpose of research while individual subjects are protected from actionable harm.

This duty to rescue approach addresses the critical role resources play in requirements of returning incidental findings. As seen by the frequency with which the authors raise the issue of resources, it is vital that an ethically sound principle be employed to appropriately find where resources fit in, as the issue of resources is frequently raised but rarely discussed in depth. A rescue-based model finds that resource restraints create an ethical ceiling by limiting the resources that can be committed toward individual subjects if it will jeopardize the ability of the researcher to complete the study. Alternatively, the duty to rescue creates a floor for disclosure obligations by finding that researchers cannot simply avoid incidental findings altogether when they discover a harm that they are in a position to alleviate.

With all of the promise that genomic research has, it is important not to lose focus on the benefits that have and will be gained by conducting this research. As such, the objectives of these studies are worthy of protection as well. The creation of a broad duty to return incidental findings, as well as search for them, could prove disastrous if this becomes a standard of care that gives rise to legal duties and potential lawsuits (Clayton and McGuire 2012). ${ }^{11}$ Certainly the resources needed to fight malpractice litigation, a large reason for bifurcating research and clinical practice, should be factored in as well (Meltzer 2006). ${ }^{12}$

Finally, a duty to rescue framework for incidental findings affords a straightforward answer to the question Gliwa et al. present: is there a duty to search for incidental findings, and if

\footnotetext{
${ }^{9}$ Michelle N. Meyer, "The Kindness of Strangers: The Donative Contract Between Subjects and Researchers and the Non-Obligation to Return Individual Results of Genetic Research,” American Journal of Bioethics 8 (2008): 45.

${ }^{10}$ Tom L. Beauchamp \& James F. Childress, Principles of Biomedical Ethics (Oxford University Press, 5th ed., 2001), 171.

${ }^{11}$ Clayton \& McGuire (2012): 473.

${ }^{12}$ Leslie A. Meltzer, “Undesirable Implications of Disclosing Individual Genetic Results to Research Participants,” American Journal of Bioethics 6 (2006): 29.
} 
not, could there be one in the future? The rescue model would suggest the answer to both questions is no. The duty to rescue applies when one finds themselves in a position to mitigate harm to another without significant risk to one’s self (Beauchamp and Childress 2001). ${ }^{13}$ However, it does not compel anyone to search out harm that they may be able to alleviate.

This distinction is relevant not only to a short term requirement but it speaks to the Gliwa et al. long-term analysis as well. The authors' focus on access to information is a patientcentered examination, where the ethical relevance is whether the subject is able to access their genomic information outside of the study. A rescue-based approach looks at access to information from the researcher perspective, placing an obligation on researchers if they access information that would lead them to believe there is a risk of harm that can be diminished (Gliwa et al. 2012). ${ }^{14}$

In this sense, the potential proliferation genomic technology in standard medicine is not relevant if a researcher has access to a subject's genomic sequence and finds an incidental finding that they believe the duty to rescue commands disclosure of. A researcher should not be able to avoid ethical obligations of reducing harm simply because a subject could access their genomic information outside of a study with relative ease. The duty to rescue applies to all moral agents (Richardson and Belsky 2004; Meyer 2008), ${ }^{15}$ and researchers are simply placing themselves in a position where they are more likely to discover genetic variants and have the capacity to prevent serious harm. Consequently, a rescue-based model will always demand a baseline obligation while maintaining no requirement to search for incidental findings regardless of the technological access to the public.

It is worth noting that this discussion surrounds what researchers are obligated to do in the conduct of genomic research. Although a rescue-based approach to incidental findings creates both a ceiling and a floor, protecting the research study and the individual subjects, respectively, there is a wide range within this spectrum where researchers can incorporate their own preferences or that of their subjects if they so choose. The key is to remind ourselves that creating a baseline that all research must be held accountable which is too high may have negative consequences. The duty to rescue framework for incidental findings makes certain that serious harm that can be alleviated will be, rather than pushing for an obligation so broad that it

\footnotetext{
${ }^{13}$ Beauchamp \& Childress (2001): 171.

${ }^{14}$ Gliwa et al. (2012): 6.

${ }^{15}$ Richardson \& Belsky (2004): 26; Meyer (2008): 45.
} 
may incentivize more anonymized research (Fryer-Edwards and Fullerton 2006) ${ }^{16}$ or, even worse, less genomic research in general. ${ }^{17}$

\section{REFERENCES}

1. Catherine Gliwa et al., Do Researchers Have an Obligation to Actively Look for Genetic Incidental Findings?, American Journal of Bioethics (2012): 1-34.

2. Maria W. Merritt, Holly A. Taylor, \& Luke C. Mullany, "Ancillary Care in CommunityBased Public Health Intervention Research,” American Journal of Public Health 100 (2010): 211-16.

3. Susan M. Wolf et al., "Managing Incidental Findings in Human Subjects Research: Analysis and Recommendations," Journal of Law, Medicine \& Ethics 36 (2008): 2-31.

4. Henry S. Richardson \& Leah Belsky, "The Ancillary-Care Responsibilities of Medical Researchers,” Hastings Center Report 34 (2004): 25-33.

5. Ellen Wright Clayton \& Amy L. McGuire, "The Legal Risks of Returning Results of Genomics Research,” Genetics in Medicine 14 (2012): 473-77.

6. Michelle N. Meyer, "The Kindness of Strangers: The Donative Contract Between Subjects and Researchers and the Non-Obligation to Return Individual Results of Genetic Research," American Journal of Bioethics 8 (2008): 44-46.

7. Tom L. Beauchamp \& James F. Childress, Principles of Biomedical Ethics (Oxford University Press, 5th ed., 2001).

8. Leslie A. Meltzer, "Undesirable Implications of Disclosing Individual Genetic Results to Research Participants,” American Journal of Bioethics 6 (2006): 28-30.

9. Kelly Fryer-Edwards \& Stephanie M. Fullerton, "Relationships with Test-Tubes: Where's the Reciprocity?,” American Journal of Bioethics 36 (2006): 36-38.

\footnotetext{
${ }^{16}$ Kelly Fryer-Edwards \& Stephanie M. Fullerton, "Relationships with Test-Tubes: Where’s the Reciprocity?,” American Journal of Bioethics 36 (2006): 37.

${ }^{17}$ Meyer (2008): 46.
} 